T. MOSER
H. GLUR $^{1}$
V. ROMANO $^{1}$
F. PIGEON
O. PARRIAUX
M.A. AHMED
T.
TRAF

T. MOSER ${ }^{1, \infty}$

H. GLUR

F. PIGEON ${ }^{2}$

M.A. AHMED

T. $\mathrm{GRAF}^{3}$

\section{Polarization-selective grating mirrors used in the generation of radial polarization}

\author{
${ }^{1}$ Institute of Applied Physics, University of Bern, Sidlerstrasse 5, 3012 Bern, Switzerland \\ ${ }^{2}$ Laboratoire TSI, Université Jean Monnet, 42000 Saint-Etienne, France \\ ${ }^{3}$ Institut für Strahlwerkzeuge, Universität of Stuttgart, Pfaffenwaldring 43, 70569 Stuttgart, Germany
}

\section{Received: 16 November 2004 / \\ Revised version: 21 February 2005 / \\ Published online: 13 April 2005 • () Springer-Verlag 2005}

ABSTRACT Two novel methods to control the polarization of laser radiation are presented. The discrimination between different polarization distributions is performed with a corrugation grating in the top high-index layer of a multilayer mirror, which couples the undesired polarization into a lossy waveguide mode of the multilayer. The generation of radially polarized radiation in a laser resonator is presented as a practical verification of the principle.

PACS 42.25.Lc; 42.25.Ja; 42.79.Fm

\section{1}

\section{Introduction}

In laser optics, controlling the polarization of light is often required. The polarization distribution of a laser beam can be controlled by means of a polarization-selective element. The simplest case is the generation of linear polarization, which can be achieved by the introduction of a Brewster plate or conventional dielectric polarizers in the optical path of the beam. The change to circular or elliptical polarization can be performed with a $\lambda / 4$ plate.

The generation of radial polarization has gained interest in recent years due to several advantages. In solid-state rod lasers, for instance, a co-axially propagating beam with radial (or tangential) polarization experiences neither depolarization nor bi-focussing because the orientation of the electric field coincides with a principal axis of the indicatrix at any point in a homogeneously pumped laser rod. Due to the avoidance of the bi-focussing, the compensation of the thermal lens can be performed more efficiently [1]. For material-processing applications high absorption of the laser radiation in the material is essential. Radially polarized beams were predicted to be up to twice more efficient for laser cutting of metals as compared to cutting with circular polarization [2]. The smallest spot ever experimentally demonstrated was shown with a radially polarized field which can be focused through a high-numericalaperture lens to a spot size significantly smaller $\left(0.16 \lambda^{2}\right)$ than

Fax: +41-31-6313765, E-mail: moser@iap.unibe.ch for linear polarization $\left(0.26 \lambda^{2}\right)$ [3]. Especially in lithography or data storage this can be an important advantage.

Different methods have been suggested to generate radial polarization to date. In a laser cavity the generation of polarized light is possible by introducing a polarization-selective element, by adapting an anisotropic transverse laser cavity geometry or by exploiting the polarizing effect of Brewsterangled optical interfaces [4-6]. According to Mushiake et al. [7] a conical mode selector ('conical Brewster plate') can be used, which has a high transmittance for the radial polarization and no transmission for the tangential components. Polarization-selective diffractive mirrors were used in $\mathrm{CO}_{2}$ lasers by Nesterov et al. [8]. These mirrors exhibit a high reflectivity for the radial polarization and direct a given fraction of the tangential polarization into diffractive orders.

In this paper two types of mirrors having a polarizationselective effect at a wavelength of $1064 \mathrm{~nm}$ based on two different mechanisms are discussed in detail: the absorbingmode mirror (AMM) and the leaky-mode mirror (LMM) [9]. The objectives of these polarization-selective mirrors are to achieve a substantial reduction of the reflection coefficient for the tangential polarization with normal incidence on the mirror without noticeably affecting the reflection coefficient of the favoured radial polarization. A detailed discussion of the principle and the characterization are presented here. It is pointed out that numerous polarization distributions can be achieved with this method such as linear, elliptical, radial, tangential, etc. Additionally, the mirror can be designed for arbitrary angles of incidence and in different variants, such as a partial reflector or a high-reflectivity mirror as it was chosen in the first demonstration reported here.

\section{2}

\section{Polarizing mechanism}

The configurations of the absorbing-mode mirror and the leaky-mode mirror are illustrated in Fig. 1. In the case of the AMM a metallic layer of 100-nm thickness and a standard multilayer manufactured by Schott Guinchard SA composed of 23 alternated high $\left(\mathrm{Ta}_{2} \mathrm{O}_{5}, n_{\mathrm{h}}=2.06\right)$ and low $\left(\mathrm{SiO}_{2}, n_{1}=1.449\right)$ refractive index layers of $\lambda / 4$ optical thickness are deposited on a fused-quartz substrate $\left(n_{\mathrm{s}}\right)$. The LMM on the other hand is composed of a standard multilayer dielectric mirror from Laseroptik $\mathrm{GmbH}$ with 29 alternated high $\left(n_{\mathrm{h}}\right)$ and low $\left(n_{1}\right)$ index layers of $\mathrm{Ta}_{2} \mathrm{O}_{5} / \mathrm{SiO}_{2}$ on a glass substrate 
a

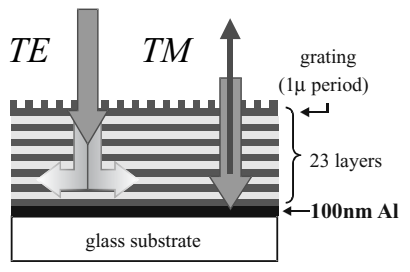

b

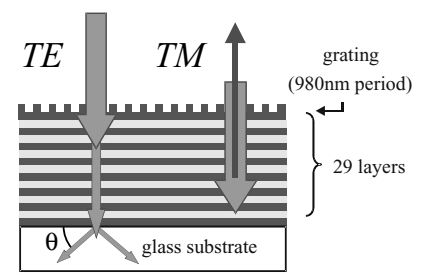

C

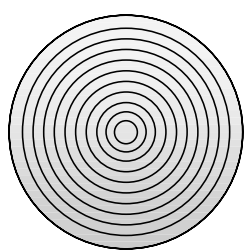

FIGURE 1 a Cross section of the absorbing-mode mirror. b Cross section of the leaky-mode mirror. c Plan view of the circular grating $\left(n_{\mathrm{s}}\right)$. The refractive indices of $\mathrm{SiO}_{2}$ and $\mathrm{Ta}_{2} \mathrm{O}_{5}$ at a wavelength of $1064 \mathrm{~nm}$ are in the case of the LMM 1.45 and 2.022, respectively.

Both mirrors include a diffraction grating, engraved in the top high-index layer by means of the electron-beam generator LION LV1 at Friedrich-Schiller-Universität Jena, Germany and Paul Scherrer Institut, Switzerland, respectively. The grating grooves have a ring shape and the complete grating has an outer diameter of $3 \mathrm{~mm}$ (AMM) and $4 \mathrm{~mm}$ (LMM), respectively. The centre part where no grating is written has a diameter of $100 \mu \mathrm{m}$ (AMM) or $150 \mu \mathrm{m}$ (LMM). The dielectric multilayer, limited by air at the top and by the metal surface (AMM) or the substrate (LMM) at the other side, represents a waveguide in which guided modes can propagate along a plane parallel to the surface due to the total internal reflection at the air side and the reflection at the metal or the substrate, respectively. The simulations to determine the optimum grating period were performed assuming a linear grating. In the following we distinguish between TE (electric field parallel to the grating lines) and TM (electric field normal to the grating lines) polarizations. The objective of the mirrors is to obtain a substantial reduction of the reflectivity for one state of polarization without affecting the orthogonal polarization. This is achieved by coupling the TE-polarized incident free-space wave to an eigenmode of the multilayer system. In Fig. 2 the multilayer system for the LMM is described in the reciprocal space, where the incoming light at normal incidence is sketched with the vertical $k$ vector $k_{0}=2 \pi / \lambda$. The dotted line shows the grating vector. The propagation vector of the coupled light is displayed as the vector $n_{\mathrm{e}} k_{0}$. The strokes represent the effective indices of the TE modes in the multilayer $\left(n_{\mathrm{e}}^{\mathrm{TE}}\right)$. The zero-order guided mode has the highest refractive index $\left(n_{\mathrm{e}}^{\mathrm{TE}_{0}}=1.729\right)$; the guided mode of the highest order has an effective index near to the refractive index of the substrate $\left(n_{\mathrm{e}}^{\mathrm{TE}_{8}}=1.451\right)$.

In the case of the LMM the existence of leaky modes [10] in addition to the guided modes is considered. The effective indices of these modes, determined by solving the dispersion equation of this leaky waveguide, are located between 1 and $n_{\mathrm{s}}$ because they undergo a total reflection at the air side and a partial reflection at the substrate side. The substrate represents a sink of power for the excited leaky modes. In a linear grating the power leakage is observed in the form of two symmetrical beams propagating along the interface between the substrate and the multilayer at angle $\theta$, as is illustrated in Fig. 1, where

$\theta=\arccos \left(\frac{n_{\mathrm{e}}}{n_{\mathrm{s}}}\right)$.

This means that the coupled energy leaks into the substrate.

Because of the presence of the metal layer in the AMM, only guided modes exist and they are characterized by refractive indices between 1 and $n_{\mathrm{h}}$. It is important to notice that the coupling of the incident radiation to a guided mode of the multilayer caused by the grating is polarization selective, since the phase-matching condition for a given wavelength (here $1064 \mathrm{~nm}$ ) and at normal incidence can only be satisfied for one polarization at a time. This implies that one can attain that only the TE polarization couples into a guided mode of the multilayer, whereas the orthogonal state of polarization (TM) is not affected by the grating but is reflected without losses by means of the multilayer mirror. The TE radiation coupled to the multilayer mode experiences losses due to the absorption in the metal layer (AMM) or through the leakage into the substrate (LMM). To achieve a coupling of the incident wave $\left(k_{0}\right)$, the $k$ vector of the grating, $k_{\mathrm{g}}=2 \pi / \lambda$, must be equal to the $k$ vector of the chosen coupled mode, $\beta=n_{\mathrm{e}} k_{0}$. For the grating period $\Lambda$ we therefore find the condition

$\Lambda=\frac{\lambda}{n_{\mathrm{e}}}$

where $\lambda$ is the wavelength and $n_{\mathrm{e}}$ the effective index of the chosen guided mode. Depending on the multilayer parameters a certain number of eigenmodes can exist. The grating

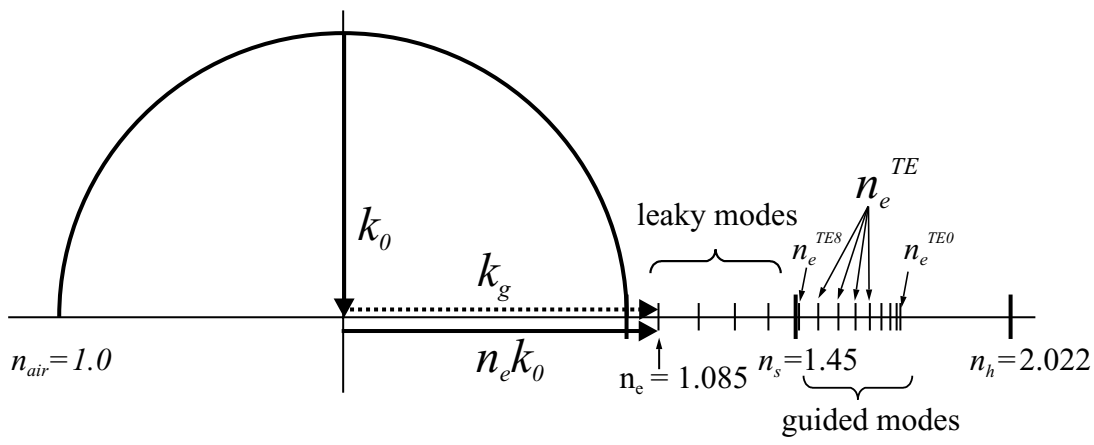

FIGURE 2 Coupling process in the LMM, described in the reciprocal space 

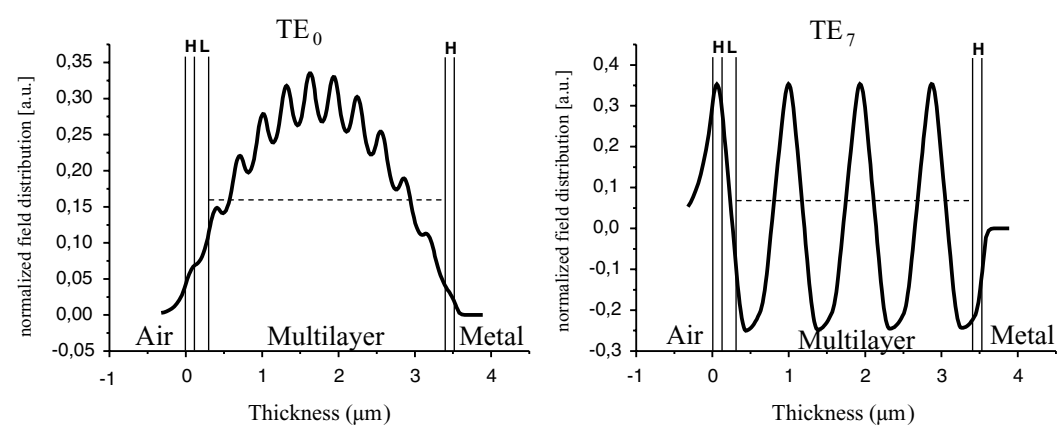

FIGURE 3 Distribution of the TE electric fields of the fundamental mode and of the three highest modes propagating in the multilayer system of the AMM ( $H$ is the high-index layer and $L$ is the low-index layer)
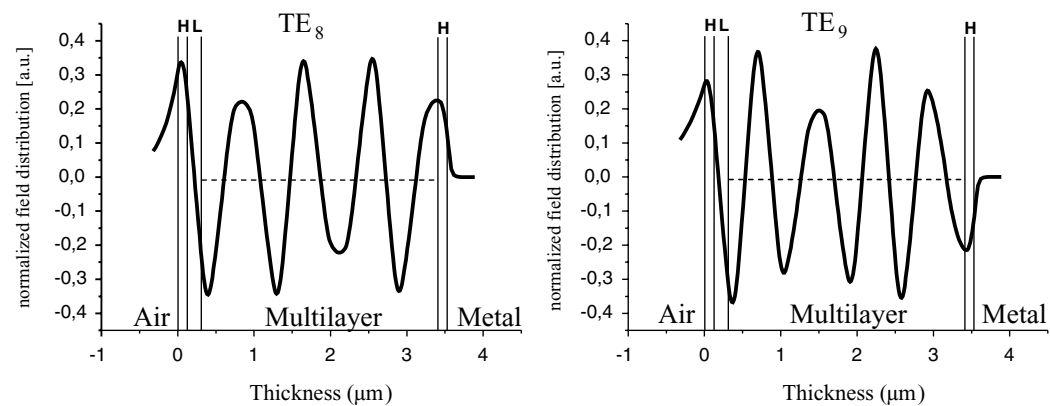

period $\Lambda$ can specifically be chosen to couple the undesired polarization to a certain guided or leaky mode of the dielectric multilayer, respectively. The type and order of mode can be chosen arbitrarily by knowing the effective index of the specific mode and choosing the grating period adequately, as seen from Eq. (2). It is mentioned that the depth of the grating affects the effective index of the coupled modes and consequently the resonance effect. Therefore, the precise etching process of the grating is a crucial point.

In order to attain efficient coupling, the normalized field overlap between the modal and the incident fields in the grating corrugation should be as high as possible. The distributions of the TE modal fields of the fundamental mode $\left(\mathrm{TE}_{0}\right)$ and of the three highest modes $\left(\mathrm{TE}_{7}, \mathrm{TE}_{8}\right.$ and $\left.\mathrm{TE}_{9}\right)$ propagating in the multilayer waveguide of the AMM are shown in Fig. 3. The mode chosen for the coupling of the incident free-space wave is a mode of high order. These high-order modes are useful for the double purpose of strong coupling and for large losses at the metal layer. Their modal fields are intense both in the grating, which guarantees efficient coupling, and at the metallic interface, which significantly affects the reflection coefficient. On the other hand the modes of low order have a field which is more concentrated in the multilayer; their absorption will be smaller and the grating strength will be weak. Additionally, a mode of high order has an effective index near to 1 and, as a result of the synchronism condition, this leads to a relatively large period, which is easier to fabricate than the corresponding period of a low-order mode.

The spectral resonance width of the coupling effect depends mainly on the grating period, the grating depth, the amplitude of the modal field, the dielectric perturbation in the grating $\left(\epsilon_{\text {grating }}-\epsilon_{\text {air }}\right)$ and the effect of the intra-guide coupling between the forward- and backward-propagating modes. In the case of the AMM the metal absorption causes an additional widening of the spectral resonance width $\Delta \lambda$ of the guided mode. The resulting spectral width of the TE reflection spectrum is $\Delta \lambda=2.5 \mathrm{~nm}$ at $80 \%$ reflectivity, which is larger than the laser gain bandwidth of Nd:YAG. For the LMM a resonance width of $\Delta \lambda=5 \mathrm{~nm}$ at $80 \%$ reflectivity was achieved. This broad resonance gives rise to a large tolerance in the insufficiently controlled multilayer system, which determines the effective index of the propagating modes. Because of the absorption in the metal layer of the AMM, the mirror will be heated and has to be cooled. A cooling mount was designed to dissipate the heat from the AMM's back side. To simplify the cooling, the metal layer could be replaced by a cooled metal block which, on the other side, will be difficult to polish to an accuracy of $\lambda / 10$ at $1064 \mathrm{~nm}$. In the case of the LMM the leaky mode is coupled into the substrate at an angle of $\Theta=48.5^{\circ}$ with respect to normal incidence and with an antireflection coating the light can be totally coupled out.

\section{$3 \quad$ Grating mirror design and fabrication}

The AMM as well as the LMM was intended to be highly reflective for the radial polarization with the option to be used as an end mirror in a laser resonator. The reflection of the undesired tangential polarization has to be decreased by a certain amount. The reflection strongly depends on the parameters of the grating. The period depth and aspect ratio of the grating as well as the number of layers of the dielectric mirror and the choice of metal are calculated with the help of an exact code based on the generalized source method [11]. Without a grating the reflection coefficient of the mirrors shown in Fig. 1 is $99.99 \%$ at normal incidence and a wavelength of $1064 \mathrm{~nm}$, as these are standard high-reflectivity laser mirrors. The period of the grating is adjusted to satisfy the synchronism condition of Eq. (2).

\subsection{Absorbing-mode mirror}

For the AMM the chosen coupled mode is of the ninth order and has an effective index of $n_{\mathrm{e}}=1.086$. The 


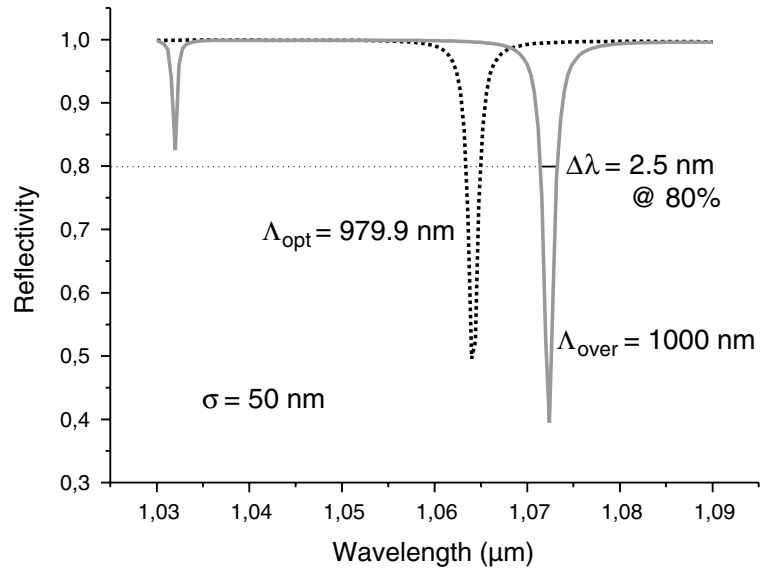

FIGURE 4 Simulation of the reflectivity of the absorbing-mode mirror: the optimum case with the resonance at $1064 \mathrm{~nm}$ (dotted line) and the version with coupling at $1072 \mathrm{~nm}$ to allow post-process trimming (solid line)

selection of a high-order mode has the advantage that the grating period $\Lambda$ is relatively large. According to Eq. (2) the grating period is $\Lambda=979.9 \mathrm{~nm}$ for $\lambda=1.064 \mu \mathrm{m}$; hence, the grating is easy to fabricate. The grating depth was chosen to be $50 \mathrm{~nm}$ where the calculations showed the best discrimination of the undesired polarization. Figure 4 illustrates the simulation of the TE and TM reflection coefficients with varying wavelength. The AMM includes a diffraction grating with a period of $979.9 \mathrm{~nm}$ and a groove depth of $50 \mathrm{~nm}$ etched into the top high-index layer $\left(\mathrm{Ta}_{2} \mathrm{O}_{5}\right)$, which leads to a dramatic decrease of the reflectivity of the undesired TE polarization at $1064 \mathrm{~nm}$. As expected the reflection coefficient of the TM polarization is affected only very slightly by the presence of the grating. The reflection coefficient at $1064 \mathrm{~nm}$ is $48 \%$ for the TE polarization, whereas it remains $99.9 \%$ for the TM polarization.

The polarizing mechanism is based on a resonant grating, which means that a small change in one of the structure parameters will affect the operation point of the device in the spectrum. The process control in multilayer technology relies upon the real-time or post-process measurement of the optical thickness of the layers. It would be highly desirable to determine the effective index of the modes from the data known from the multilayer supplier. This is however not straightforwardly possible because of the small uncertainties of the fabrication process. Due to this insufficient control of the multilayer parameters (refractive index as well as thickness of the layers), the grating period was chosen in such a way that a shift of the polarization effect could be achieved by post-processing (trimming). In the trimming process, the top layer (grating included) is etched down in thickness. In this way, the effective index of the chosen guided mode in the multilayer decreases and therefore the wavelength where the effect should appear can be lowered. Consequently, the period was chosen to be $1 \mu \mathrm{m}$ (instead of $979.9 \mathrm{~nm}$ ), whereon the coupling at normal incidence for the TE polarization would be found at a wavelength of $1.072 \mu \mathrm{m}$; the effective index of the guided mode was already mentioned to be $n_{\mathrm{e}}=1.086$. The depth was chosen to be $50 \mathrm{~nm}$. The simulation of the reflectivity is shown in Fig. 4 for the optimum case (with the effect at $1064 \mathrm{~nm}$ but no possibility for post-processing) and for the case with a longer period to be trimmed by post-process etching. A shift of the resonance wavelength by $8 \mathrm{~nm}$ could be achieved by etching away about $20 \mathrm{~nm}$ of the top layer.

The grating with a period of $1 \mu \mathrm{m}$ was written into the last high-index layer of the mirror by means of the electron-beam generator LION LV1 and then etched by Reactive Ion Beam Etching (RIBE) at Friedrich-Schiller-Universität Jena. Figure 5a shows the relief structure scanned with an atomic force microscope (AFM). A picture of the absorbing-mode mirror with the circular grating situated in the centre and an additional linear grating for measurement purposes is shown in Fig. 5b.

\subsection{Leaky-mode mirror}

For the leaky-mode mirror the chosen mode to couple the TE polarization is a leaky mode of the third order. Its effective index is 1.085 and therefore the grating period has to be $980 \mathrm{~nm}$. Figure 6 illustrates the simulation of the reflectivity for the TE and TM polarizations versus wavelength for the LMM comprising a diffraction grating with a period of $980 \mathrm{~nm}$ and a groove depth of $70 \mathrm{~nm}$ etched in the top high-index layer $\left(\mathrm{Ta}_{2} \mathrm{O}_{5}\right)$. As expected the reflection coefficient of the TM polarization, $99.3 \%$, is affected only very slightly by the presence of the grating, whereas for the TE polarization the reflection coefficient at $1064 \mathrm{~nm}$ is decreased to $76.6 \%$. The spectral width of the TE reflection spectrum at a reflectivity of $85 \%$ is $8.5 \mathrm{~nm}$. This wide spectral range has the advantageous consequence of being a more tolerant solution regarding the multilayer parameters than the AMM. Due to this the trimming process could be skipped and the mirror was straightforwardly manufactured according to the simulated dimensions.

As in the case of the AMM, the grating was written on a small area of the deposited substrate by means of the a

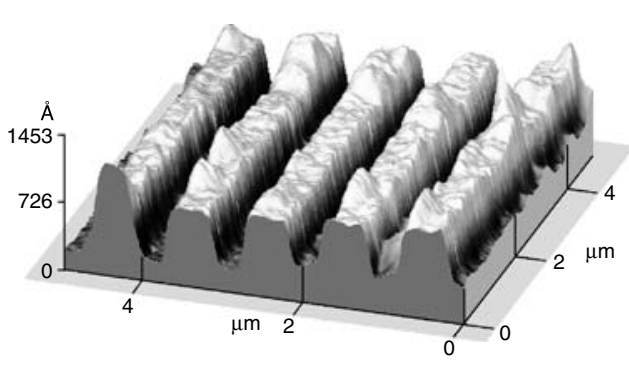

b

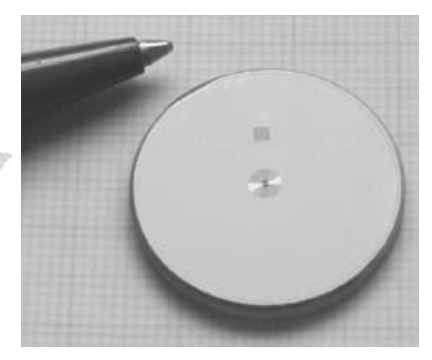

FIGURE 5 a AFM picture of the absorbing-mode mirror. b The absorbing-mode mirror 


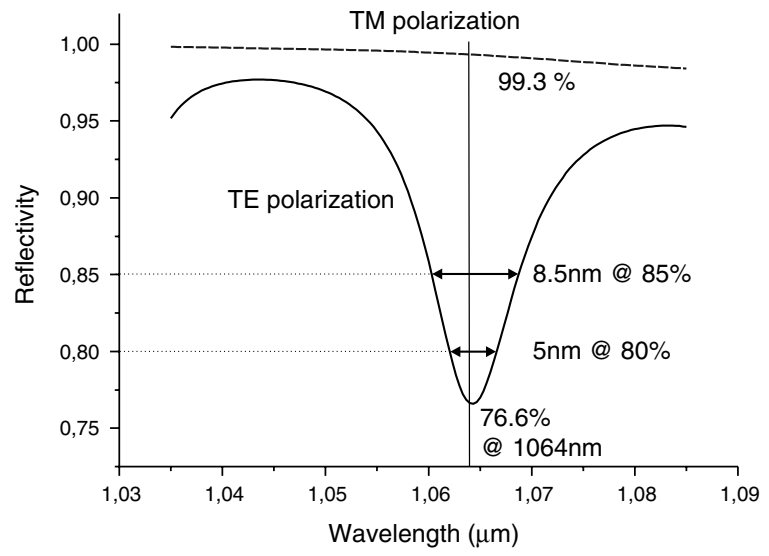

FIGURE 6 Modelling of the reflection coefficient for the LMM with a diffraction grating of 980-nm period and 70-nm groove depth

electron-beam generator LION LV1 at PSI-Zurich and then etched by RIBE down to a depth of $80 \mathrm{~nm}$ and an aspect ratio of about $50 \%$ as measured by an AFM (see Fig. 7a) at Friedrich-Schiller-Universität Jena. Figure $7 \mathrm{~b}$ shows the leaky-mode mirror with the circular grating situated in the centre and an additional linear grating for diagnostic purposes. The latter was used to determine the grating period with a setup under the Littrow condition and to measure the reflectivity with a linearly polarized laser beam. The effective indices of the guided modes were measured by coupling a linearly polarized laser beam at different angles into the referring modes, whereon the reliability of the supplier's data was confirmed.

\section{4}

\section{Characterization}

The characterization of the polarizing effect of the AMM was made by placing the mirror in the spectroscopic setup shown in Fig. 8. Similar to the simulation assuming the ring-shaped grating to work as a composition of infinite linear gratings, the characterization of the mirror was done on a linear grating. The beam of a white-light source (with a non-uniform irradiation over the spectral range) was linearly polarized by an additional polarizer and sent through a 1mm-diameter diaphragm to obtain a beam smaller than the grating. At normal incidence the light is sent onto the linear test grating, which has a diameter of $1.5 \mathrm{~mm}$.

A beam splitter redirects the reflected beam to the entrance of the spectrometer. A germanium photodetector operating at

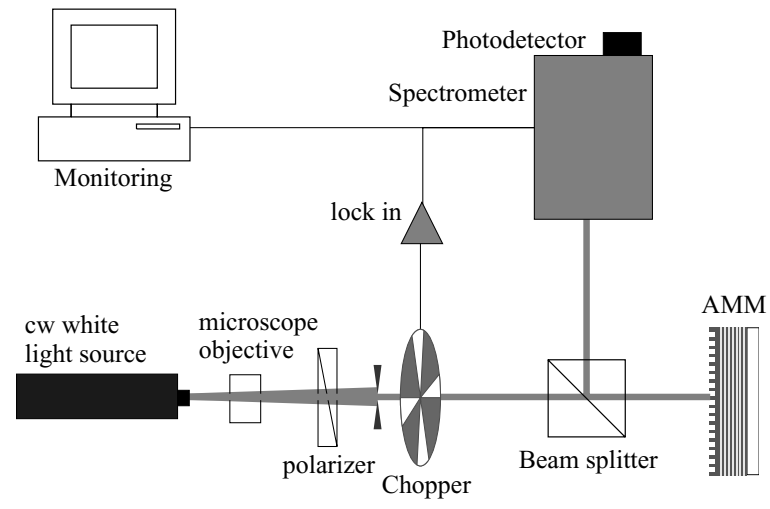

FIGURE 8 Spectral measurement experimental setup

$77 \mathrm{~K}$ in combination with a lock-in amplifier detects the optical power reflected by the polarization-selective multilayer grating mirror as a function of the wavelength. With this setup the spectral dependence of the reflection of light could be measured. The white light is not perfectly linearly polarized ( $\sim 1: 100)$. But this does not affect the position of the resonance dip; it only affects the discrimination of the polarization and, therefore, limits the determination of the reflectivity to $1 \%$ inaccuracy. Before the trimming of the absorbing-mode mirror there was a substantial reduction of the reflection coefficient at $1068.7 \mathrm{~nm}$, slightly above the desired wavelength of $1064 \mathrm{~nm}$ for the TE polarization as shown in Fig. 9a. The difference between the simulated dip at $1072 \mathrm{~nm}$, shown in Fig. 6, and the measured effect at $1068.7 \mathrm{~nm}$ can be traced back to the fact that the etching process was too strong, which resulted in a grating depth of $\sim 70 \mathrm{~nm}$ instead of the specified $50 \mathrm{~nm}$ by the simulation. The fact that the resonance effect is found at a higher wavelength than desired permits a trimming step consisting of a short homogeneous etching to lower the coupling wavelength to $1064 \mathrm{~nm}$ by decreasing the effective index of the desired guided mode. Figure $9 \mathrm{~b}$ shows the reflection spectrum after the trimming operation for both polarizations. The dip in the reflectivity spectrum was lowered to the desired $1064 \mathrm{~nm}$.

The reflectivity was determined with a linearly polarized $\mathrm{Nd}$ :YAG laser beam incident on the linear test grating and was found to be about $97 \%$ for the TM polarization and about $50 \%$ for the TE polarization. The leaky-mode mirror was characterized with the measurement of the effective indices of the guided modes. A linearly polarized helium-neon laser was a

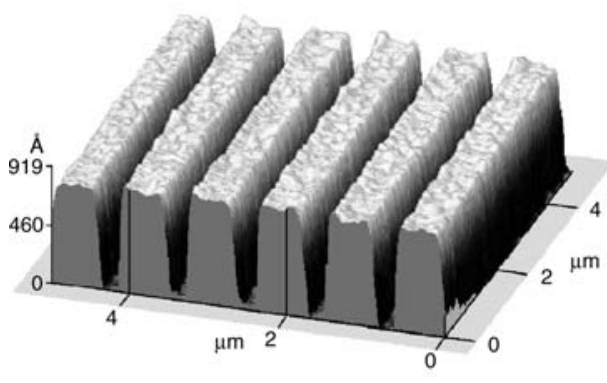

b

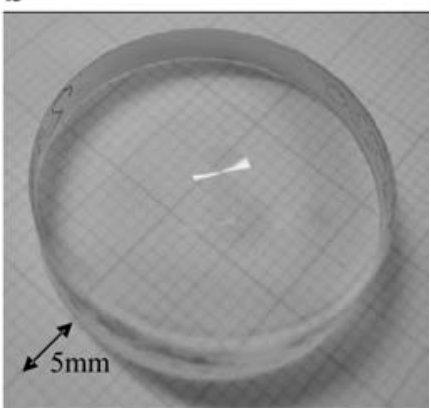

FIGURE 7 a AFM picture of the leaky-mode mirror. b The leaky-mode mirror 

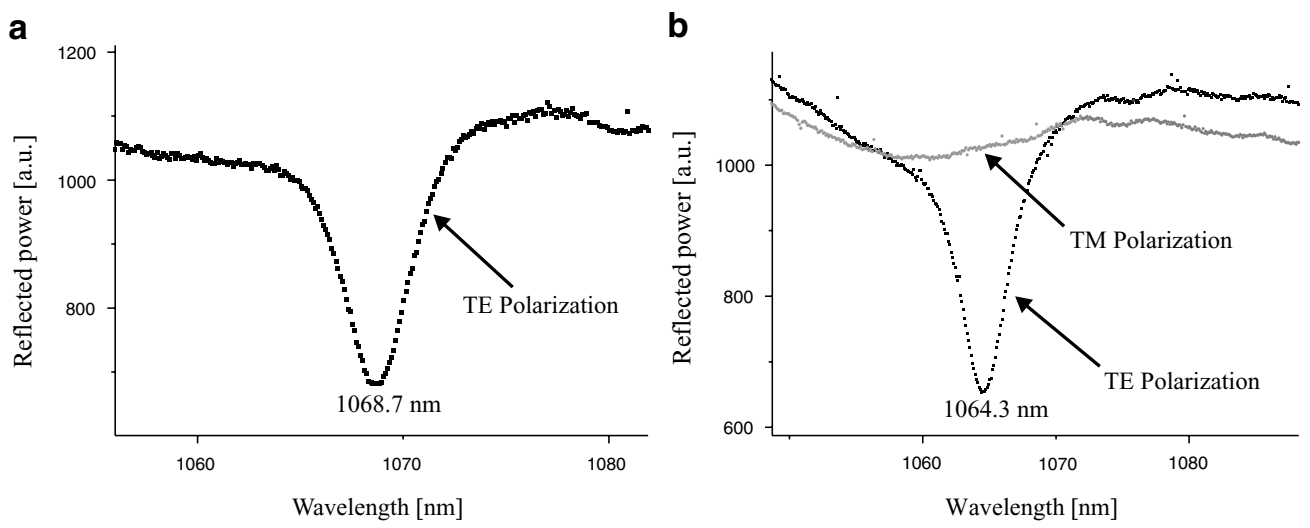

FIGURE 9 Experimental reflection spectrum of the TE polarization $\mathbf{a}$ before trimming and $\mathbf{b}$ after trimming

pointed at the linear grating at different incident angles. At certain angles a clear resonance was visible, which indicated the coupling of the incident light into a guided mode. With the knowledge of the incident angle the effective index of the said mode was determined. The experimental data could then be compared with the data obtained by the modelling with the supplier's data, which showed slight deviations from the simulated data of the layer thickness and the refractive index. But, thanks to the wide spectral width, the mirror still performed as desired. The reflectivity was measured to be $97 \pm 2 \%$ for the TM polarization and around $70 \%$ for the TE polarization.

\section{5}

\section{Radial polarization}

Radial polarization has attracted attention in recent years $[2,3,6]$. Here we report the generation of radial polarization in a Nd:YAG laser with the use of the described dielectric mirrors based on the resonant polarizing effect. Experimental results of the AMM were published in [9]; further experiments have not been performed due to the limits imposed by the heating of the mirror that was caused by depolarization of the laser beam and the consequent absorption in the aluminium layer at high powers. The following report is therefore restricted to the experimental results on the generation of radially polarized radiation with the leaky-mode mirror.

In earlier reports it was shown that a radially symmetric pump distribution is essential for the generation of radial polarization. In Moser et al. [9] a transversally diode pumped $\mathrm{Nd}$ :YAG rod laser was used for the experiments. The nonradially symmetric pump distribution (sixfold, Fig. 10a) was
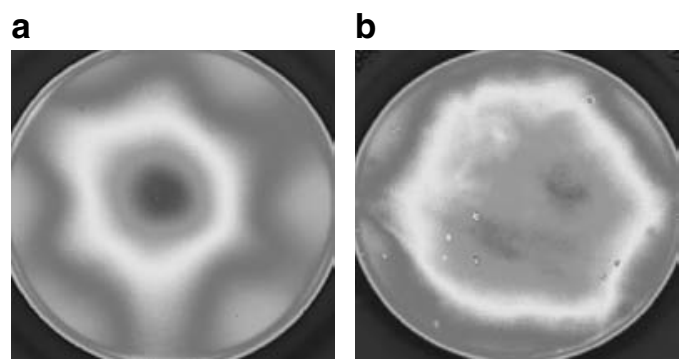

FIGURE 10 Fluorescence image of the laser cavity used taken through a pinhole in the far field. a Non-radially symmetric distribution, b partially diffuse pumped cavity

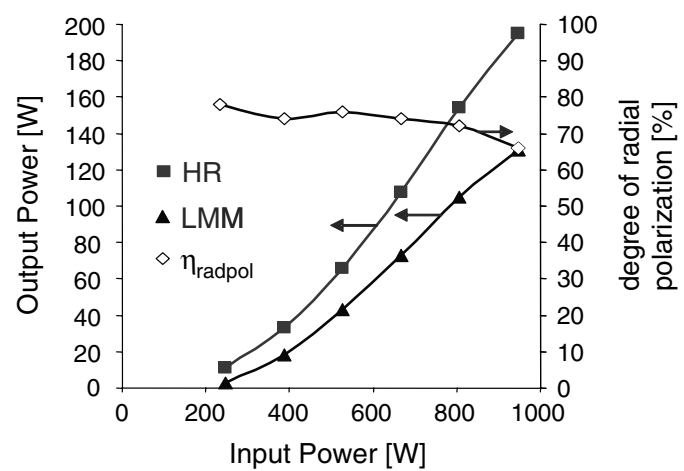

FIGURE 11 Output power of the unpolarized laser (HR) and the laser with radial polarization (LMM) and the degree of polarization $\left(\eta_{\text {radpol }}=\right.$ $\left.P_{\text {rad }} / P_{\text {out }}\right)$ versus the optical input power

responsible for severe aberrations at high pump powers for the radially polarized light also. With the use of an aperture, whereby only the centre part of the laser rod with a homogeneous pump light distribution is used, it is possible to generate radially polarized beams with a very high purity but with moderate efficiency of the laser.

In the newest experiments the leaky-mode mirror was used in a diffuse diode-pumped Nd:YAG rod laser with a more radially symmetric pump distribution, Fig. 10b. The laser rod had a diameter of $4 \mathrm{~mm}$ and the symmetric resonator had a length of $210 \mathrm{~mm}$. The polarization-selective element ensures that once the laser has been adjusted (with respect to maximum output power), the emitted light is radially polarized over the whole stability range of the laser resonator. Output powers up to $130 \mathrm{~W}$ were achieved in this configuration. Figure 11 shows the output power and the degree of polarization versus input power.

The degree of polarization was determined by calculating the angular correlation of the measured intensity distribution after a linear polarizer with the functions $\cos ^{2}(\varphi)$ and $\sin ^{2}(\varphi)$, where $\varphi$ is the angle between the radial vector and the axis of the polarizer. To verify the radial symmetry, which is a precondition of the calculation, the method was applied once in the horizontal and once in the vertical direction. In our definition the degree of radial polarization represents the fraction of radially polarized light divided by the total power. With increasing pump power the polarization degree decreases from 

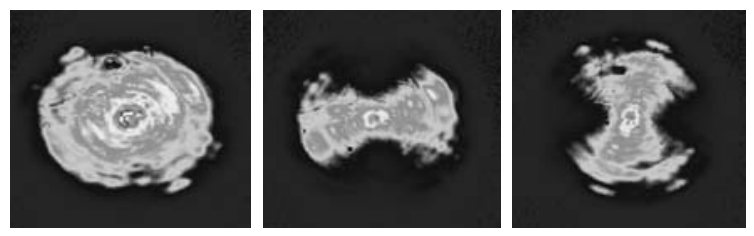

19W
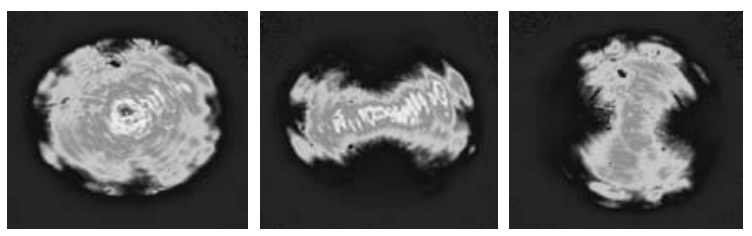

$43 \mathrm{~W}$
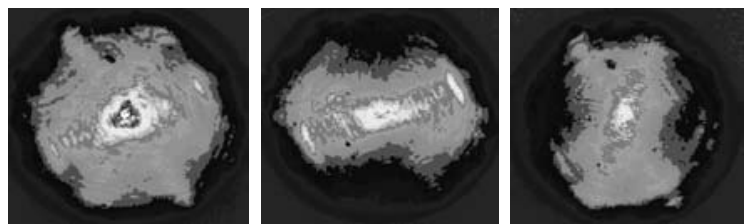

FIGURE 12 Radially polarized laser beam with different output powers. Recorded behind the LMM without polarizer, and through a polarizer adjusted horizontally and vertically

A

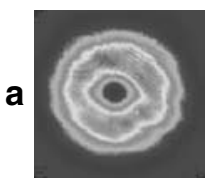

b

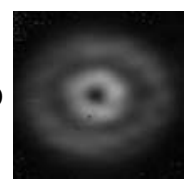

B
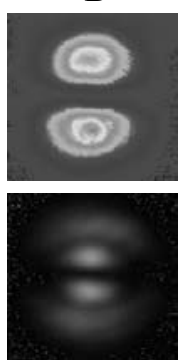
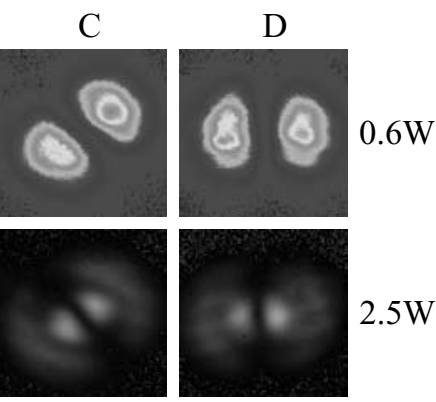

FIGURE 13 The beam recorded behind the LMM for different output powers. A No polarizer, B, C, D through a polarizer adjusted B vertically, C at $45^{\circ}$, D horizontally

an initial value of $80 \%$ to about $65 \%$ at high pump power, Fig. 12. With a standard HR mirror the laser has a moderate optical efficiency of about $20 \%$. The efficiency for radial polarization was measured to be up to $14 \%$ with the LMM. The additional losses have two major origins: firstly, the overlap of the low-order radially polarized modes and the gain profile is reduced as compared to the unpolarized case because the radial polarization has a zero point in the centre. Secondly, the light passing through the rod still undergoes some depolarization due to residual inhomogeneities of the pumping distribution and will therefore couple out at the LMM. Additionally, our first ever LMM prototype has a reflectivity of slightly less than $100 \%$.

With a longer resonator $(610 \mathrm{~mm})$ the beam quality can be improved and consequently the degree of radial polarization can be increased at low powers. Figure 13a shows the fundamental doughnut mode generated with a high radial polarization purity (90\%). In Fig. 13b, where the second order of the shaped doughnut modes starts oscillating, $90 \%$ of the light is still radially polarized.

It is important to note that the polarization distribution is also radial in the far field. As already described above, radial polarization which was depolarized while passing twice through the rod and unpolarized modes oscillating in the centre of the beam leak in the LMM.

\section{6}

\section{Conclusion}

In summary, we have developed and demonstrated two polarization-selective mirrors that operate at normal incidence and which are based on two different filtering mechanisms. The characterization of the mirror properties showed good agreement with the simulations. The guided and leaky modes of the multilayer system are clearly seen when the incident TE-polarized light is coupled to them. In the case of the LMM a very tolerant design with respect to irregularities of the multilayer and the grating structure was demonstrated. The functionality of the absorbing-mode mirror was shown at low powers. With the LMM further experiments were performed, as it is better suited for high-power applications. Fundamental and higher-order radially polarized ring modes were demonstrated experimentally with polarization purities exceeding $90 \%$. Output powers beyond $100 \mathrm{~W}$ of a radially polarized Nd:YAG laser with a degree of polarization exceeding $70 \%$ were shown. The maximum power was limited by the available pump power. A moderate decrease of the efficiency of the laser is caused by the depolarization in the rod due to a nonradially symmetric pumping distribution and the unwanted oscillation of the unpolarized modes in the centre. Future efforts have to be concentrated on the design of a laser cavity with a pure radially symmetric pump distribution in order to avoid the depolarization in the laser rod.

ACKNOWLEDGEMENTS This work was supported by the CTI of the Swiss Government. The authors acknowledge the contribution of Dr. J. Fuchs, Friedrich-Schiller-Universität Jena and Dr. L. Heyderman, Paul Scherrer Institut in grating fabrication.

\section{REFERENCES}

1 E. Wyss, M. Roth, T. Graf, H.P. Weber, IEEE J. Quantum Electron. 38(12), 1620 (2002)

2 V.G. Niziev, A.V. Nesterov, J. Phys. D: Appl. Phys. 32, 1455 (1999)

3 R. Dorn, S. Quabis, G. Leuchs, Phys. Rev. Lett. $91(23), 233901$ (2003)

4 D. Pohl, Appl. Phys. Lett. 20(7), 266 (1997)

5 R. Oron, S. Blit, N. Davidson, A.A. Friesem, Z. Bomzon, E. Hasman, Appl. Phys. Lett. 77(21), 3322 (2000)

6 I. Moshe, S. Jackel, A. Meir, Opt. Lett. 28(10), 807 (2003)

7 Y. Mushiake, K. Matsumura, N. Nakajima, Proc. IEEE 60, 1107 (1972)

8 A.V. Nesterov, V.G. Niziev, V.P. Yakunin, J. Phys. D: Appl. Phys. 32, 2871 (1999)

9 T. Moser, M.A. Ahmed, F. Pigeon, O. Parriaux, E. Wyss, Th. Graf, Laser Phys. Lett. 1(5), 234 (2004)

10 J.D. Décotignie, O. Parriaux, F.E. Gardiol, Int. J. Electron. Commun. (AEÜ) 35(5), 201 (1981)

11 A.V. Tishchenko, Pure Appl. Opt. 7, 1425 (1998) 